\title{
Epithelium-specific Ets transcription factor- 1 acts as a negative regulator of cyclooxygenase-2 in human rheumatoid arthritis synovial fibroblasts
}

Chan-Mi Lee ${ }^{1,2}$, Sahil Gupta ${ }^{3,4}$, Jiafeng Wang ${ }^{3,6}$, Elizabeth M. Johnson ${ }^{7}$, Leslie J. Crofford ${ }^{7}$, John C. Marshall ${ }^{3,4,5}$, Mohit Kapoor ${ }^{2,8}$ and $\operatorname{Jim~Hu} u^{1,2,4^{*}}$

\begin{abstract}
Background: Rheumatoid arthritis (RA) is characterized by excessive synovial inflammation. Cyclooxygenase-2 (COX2) is an enzyme that catalyzes the conversion of arachidonic acid (AA) into prostaglandins. Epithelium-specific Ets transcription factor-1 (ESE-1) was previously demonstrated to upregulate COX-2 in co-operation with nuclear factor kappa B (NFKB) in macrophages and chondrocytes. However, the role of ESE-1 in RA pathology has remained unclear. In this study, we aimed to elucidate the relationship between ESE-1 and COX-2 in RA synovial fibroblasts (RASFs) using a HD-Ad-mediated knockdown approach.
\end{abstract}

Results: ESE-1 and COX-2 were induced by IL-1 $\beta$ in RASFs that corresponded with an increase in PGE 2 . Endogenous levels of ESE- 1 and COX-2 in human RASFs were analyzed by RT-qPCR and Western blot, and PGE 2 was quantified using competitive ELISA. Interestingly, knockdown of ESE-1 using helper-dependent adenovirus (HD-Ad) led to a significant upregulation of COX-2 at a later phase of IL-1 $\beta$ stimulation. Examination of ESE-1 intracellular localization by nuclear fractionation revealed that ESE-1 was localized in the nucleus, occupying disparate cellular compartments to NFKB when COX-2 was increased. To confirm the ESE-1-COX-2 relationship in other cellular systems, COX-2 was also measured in SW982 synovial sarcoma cell line and ESE-1 knockout (KO) murine macrophages. Similarly, knockdown of ESE-1 transcriptionally upregulated COX-2 in SW982 and ESE-1 KO murine macrophages, suggesting that ESE-1 may be involved in the resolution of inflammation.

Conclusion: ESE-1 acts as a negative regulator of COX-2 in human RASFs and its effect on COX-2 is NFkB-independent.

Keywords: Rheumatoid arthritis, ESE-1, COX-2, Inflammation, Synovial fibroblasts, Helper-dependent adenovirus, Prostaglandins

\section{Background}

Rheumatoid arthritis (RA) is a systemic autoimmune disease characterized by the progressive destruction of the joints due to excessive inflammation in the synovium, which can lead to deformities and loss of joint function

\footnotetext{
*Correspondence: jim.hu@utoronto.ca

1 SickKids Research Institute, Program in Physiology and Experimental Medicine, The Hospital for Sick Children, Peter Gilgan Centre for Research and Learning, 9th floor, 686 Bay Street, Toronto, ON M5G 0A4, Canada Full list of author information is available at the end of the article
}

in severe cases. Inflammation in the RA synovial tissue is perpetrated by the production of inflammatory cytokines and secreted mediators from infiltrating immune cells and activated synovial fibroblasts [1]. Prostaglandins (PGs) are key mediators responsible for RA symptoms of pain and swelling [2]. Synthesis of PG requires conversion of arachidonic acid released from cell membranes to prostaglandin $\mathrm{H}_{2}\left(\mathrm{PGH}_{2}\right)$, the critical step of which is catalyzed by cyclooxygenase- 2 (COX-2), also known as the $\mathrm{PGH}_{2}$ synthase. $\mathrm{PGH}_{2}$ is further metabolized to bioactive 
forms such as $\mathrm{PGE}_{2}$, prostacyclin, prostaglandin $\mathrm{D}_{2}$, and prostaglandin $F_{2 \alpha}$, by their respective synthases in different cell types $[2,3]$. COX-2 is highly expressed in the RA synovial lining due to the persistent presence of proinflammatory cytokines such as IL- $1 \beta$, TNF- $\alpha$, and IL- 6 , and is a key biosynthetic enzyme regulating PG production in the synovium $[4,5] . \mathrm{PGE}_{2}$ is the major PG that is generated by chondrocytes and synovial fibroblasts [6], and clinical responses to non-steroidal anti-inflammatory drugs (NSAIDs) have been shown to correlate with reduced levels of $\mathrm{PGE}_{2}$ in the synovial fluid $[7,8]$. COX-2 inhibitors such as celecoxib effectively control arthritis symptoms [8].

COX-2 gene activation is complex and employs numerous regulatory factors specific to different stimuli, as exemplified by the COX-2 promoter which contains two NFkB motifs, two activator protein 1 (AP-1) sites and two cAMP-response elements (CREs) among others [9]. Several Ets factors have also been shown to regulate COX-2 expression, including Ets-1 [10], Pea3 [11] and PU.1 [12], and Elk1 [13] in different tissue contexts. Ets family of transcription factors are characterized by the highly conserved E26 transformation-specific (Ets) DNA binding domain, which recognizes GGAA/T core consensus sequence within the promoter and enhancer regions of target genes [14]. Unlike most Ets factors which are expressed in hematopoietic cells, however, a subgroup of Ets proteins called epithelium-specific Ets factors (ESE's) has epithelium-restricted expression pattern under basal conditions. Interestingly, ESE-1, the prototype of ESE subfamily, is highly sensitive to inflammatory stimulation [15], where it was found to be expressed in the human RA synovial tissue [16]. It was also transcriptionally upregulated by proinflammatory stimuli such as IL- $1 \beta$, TNF- $\alpha$, or LPS in the resident cell types including synovial fibroblasts, chondrocytes, osteoblasts, and macrophages, typically displaying a peak expression between $2-6 \mathrm{~h}$ and dissipation by $24 \mathrm{~h}$ in most cells [16]. ESE-1, or Elf3 in mice, was similarly found to transactivate COX-2 promoter in murine macrophages and human chondrocytes in cooperation with NFkB [17], suggesting its critical role in RA pathogenesis. However, initial analyses had revealed ESE-1 to be predominantly expressed in the cytoplasm of the cells [16], leaving discrepancies in how it might function as a transcription factor in situ. Additionally, the prolonged expression of ESE-1 mRNA in RASFs beyond $24 \mathrm{~h}$ of IL-1 $\beta$ stimulation unlike in other cell types has left the relationship between ESE-1 and COX-2 in RASFs elusive. As RASFs and synovial macrophages are prominent cell types present in the terminal layer of the hyperplastic synovial tissue which secrete inflammatory cytokines and matrix-degrading enzymes [18, 19], elucidation of the role of ESE-1 in COX-2 regulation is important to gain better understanding of the molecular events that occur in RA synovial tissues.

Previous studies primarily focused on investigating functional significance of ESE-1 by ESE-1 overexpression, where ESE-1 cDNA was transfected into cell lines along with luciferase constructs to investigate the transactivation of ESE-1 on its target genes. However, ectopic gene expression can lead to supraphysiological levels of the gene of interest, as well as cell toxicity from the transfection procedure itself. Also, overexpression by transfection may not accurately reflect the temporal behaviour of a protein, and may thus lead to artificial interaction or co-localization of proteins that do not normally co-exist under physiological conditions. ESE-1 overexpression could also have accompanied co-induction of its other target genes, giving rise to confounding results. Therefore, we sought to elucidate the relationship between ESE-1 and COX-2 in human RASFs using a knockdown approach with helper-dependent adenoviral (HD-Ad) vector, which has all of the viral genes removed to render it much less immunogenic than conventional adenoviruses [20, 21], and in Elf3 knockout mouse bone marrow-derived macrophages (BMDMs) to avoid side effects from transfection- or transduction-mediated gene manipulation. In this study, we show for the first time that ESE-1 negatively regulates COX-2 in human RASFs.

\section{Methods \\ Reagents}

DMEM, RPMI, fetal bovine serum (FBS) and L-glutamine were purchased from Gibco Life Technologies Ltd., Burlington, Ontario, Canada. Penicillin/streptomycin, phosphate buffered saline (PBS) were from Wisent, St. Bruno, Quebec, Canada. Human recombinant IL-1 $\beta$ was product of R\&D Systems (Minneapolis, MN, USA), and LPS endotoxin (Escherichia coli, serotype O128:B12) and DEAE-Dextran hydrochloride of Sigma (Oakville, Ontario, Canada), while murine IL-4 was from Peprotech, Quebec, Canada. Antibodies used in this study were: COX-2 rabbit polyclonal antibody from Thermo Fisher Scientific (Burlington, Ontario, Canada), and COX-2 (C-20) goat polyclonal, NFkB p65 (C-20), p50 (H-119) and Lamin A (H-102) rabbit polyclonal antibodies from Santa Cruz (Dallas, TX, USA). ESE-1 rabbit monoclonal antibody was produced in our laboratory in collaboration with Epitomics, Burlingame, CA, USA [22]. Hsp90 rabbit polyclonal and $\beta$-actin mouse monoclonal antibodies were purchased from Cell Signaling Technology (Whitby, Ontario, Canada).

\section{Preparation of RASFs}

Synovial tissues were obtained at the time of joint replacement surgery from patients with RA who fulfilled 
the revised American Rheumatism Association criteria for this disease [7]. Experiments were carried out according to a protocol that was approved by the Institutional Review Board in Vanderbilt University, Nashville, TN, and patient informed consent was obtained. RASF were prepared as previously described [9]. Briefly, minced synovial tissues were digested overnight with $1 \mathrm{mg} / \mathrm{ml}$ collagenase (Type I, Sigma, St. Louis, MO, USA) in DMEM in a humidified $5 \% \mathrm{CO} 2$ incubator at $37{ }^{\circ} \mathrm{C}$ and the isolated cells were cultured in $175 \mathrm{~cm}^{2}$ culture flasks in DMEM supplemented with $20 \%$ FBS, L-glutamine $(2 \mathrm{mM})$, penicillin $(100$ units $/ \mathrm{ml})$ and streptomycin $(100 \mu \mathrm{g} / \mathrm{ml})$. At greater than $95 \%$ confluency, the adherent RSF were passaged by digestion with $0.05 \%$ trypsin/EDTA and used for cell culture experiments.

\section{Cell culture}

Human synovial sarcoma and lung adenocarcinoma cell lines SW982 and A549 were obtained from American Type Culture Collection, Rockville, MD, and were cultured in DMEM supplemented with $10 \%$ FBS and $100 \mathrm{IU} / \mathrm{mL}$ penicillin, and $100 \mu \mathrm{g} / \mathrm{mL}$ streptomycin in $5 \% \mathrm{CO}_{2}$ at $37{ }^{\circ} \mathrm{C}$. Cells were starved in serum-deprived medium containing $0.5 \% \mathrm{FBS}$, in which transduction and cytokine stimulation were also performed.

\section{Infection of cells with helper-dependent adenovirus (HD-Ad)}

$E S E-1$ gene was knocked down in human synovial fibroblasts and SW982 cells using shRNA helper-dependent adenoviral vector expressing two shRNAs prepared as previously described [23, 24], with added modifications from [25]. Briefly, cells were seeded at 100,000 cells per well in growth medium on 6-well plates a day prior to transduction, and 5000 virus DNA particles per cell equivalent of $100 \mathrm{MOI}$ were complexed with $520.5 \mathrm{ng}$ DEAE-Dextran by incubation for $30 \mathrm{~min}$ at room temperature in $0.5 \%$ FBS DMEM. The DEAE-virus mixture was added to cells by replacing the growth medium. C4HSU empty vector was used as control. The cells were incubated for $2 \mathrm{~h}$ in a $5 \% \mathrm{CO}_{2}$ at $37{ }^{\circ} \mathrm{C}$, after which $20 \%$ FBS DMEM was added to achieve a final concentration of $10 \%$ FBS. The cells were then incubated for additional $48 \mathrm{~h}$, and the medium was removed and replaced with $0.5 \%$ FBS DMEM for $24 \mathrm{~h}$ starvation before being stimulated with $10 \mathrm{ng} / \mathrm{mL}$ IL-1 $\beta$.

\section{RNA isolation and real-time quantitative PCR (RT-qPCR)} Total RNA was isolated using GE Illustra RNAspin Mini Kit (GE Healthcare Life Sciences, Baie-D'Urfe, Quebec) as per manufacturer's instructions. For real-time quantitative PCR, after spectrophotometry quantification, $1 \mu \mathrm{g}$ of RNA was reversed transcribed in a final volume of $20 \mu \mathrm{L}$ using Superscript VILO Mastermix with Superscript III (Invitrogen, Carlsbad, CA) and the resulting cDNA template $(10 \mathrm{ng}$ ) was used for qPCR reaction using Power SYBR Green PCR Master Mix from Life Technologies (Burlington, Ontario, Canada). ViiA ${ }^{\mathrm{TM}} 7$ RealTime 384-well PCR System from Life Technologies was used for the amplification and analysis. For relative $\Delta \Delta \mathrm{Ct}$ quantification, qPCR signals were normalized using GAPDH and fold changes were calculated according to Livak and Schmittgen [26]. The primer sequences used for human and mouse samples are provided in Additional file 1: Table S1.

\section{Cytoplasmic nuclear fractionation and Western blot}

Nuclear and cytoplasmic extracts were prepared from human RASFs by nuclear/cytoplasmic separation as previously described [27]. In summary, cells grown in $10 \mathrm{~cm}$ dishes were washed twice with ice-cold PBS, and pelleted cells were resuspended in $900 \mu \mathrm{L}$ of hypotonic buffer containing $0.1 \%$ NP-40 in PBS containing protease inhibitors (Roche; Mississauga, Ontario, Canada) and triturated five times, after which they were immediately centrifuged at $500 \times g$ at $4{ }^{\circ} \mathrm{C}$. The supernatant was collected and designated as the cytoplasmic extract, while the pellet was washed once with $1 \mathrm{~mL}$ of $0.1 \% \mathrm{NP}-40$ PBS buffer, re-centrifuged, and lysed in $180 \mu \mathrm{L} 6 \times$ SDS sample buffer [2\%(w/v) SDS, $58.3 \mathrm{mM}$ Tris- $\mathrm{HCl}(\mathrm{pH}$ 6.8), $6 \%(\mathrm{v} / \mathrm{v})$ glycerol, $5 \%(\mathrm{v} / \mathrm{v}) 2-\beta$-mercaptoethanol, $0.02 \%(\mathrm{w} / \mathrm{v})$ bromophenol blue] and was designated as the nuclear extract. The nuclear extract was sonicated at Level 2 on Misonix 3000 sonicator for 5-10 s. Lysates were separated by electrophoresis on $10 \%$ SDS-PAGE gel, and transferred to nitrocellulose membrane (Amersham; GE Healthcare, Mississauga, Ontario, Canada). Membranes were blocked with $5 \%(\mathrm{w} / \mathrm{v})$ nonfat milk in TBST $(50 \mathrm{mM}$ Tris-Cl pH 7.5, $150 \mathrm{mM} \mathrm{NaCl}, 0.05 \%$ Tween-20) for $1 \mathrm{~h}$ at room temperature and probed for ESE-1 (1:3000), COX-2 (1:500), Hsp90 (1:1000), $\beta$-actin (1:4000), or Lamin A (1:500) overnight at $4{ }^{\circ} \mathrm{C}$. Protein signals were detected with HRP-conjugated secondary antibodies at a dilution of 1:4000 using ECL Western blotting detection system (Amersham Pharmacia Biotech, Baie-D'Urfe, Quebec, Canada).

\section{Prostaglandin $\mathrm{E}_{2}\left(\mathrm{PGE}_{2}\right)$ quantification}

$\mathrm{PGE}_{2}$ was quantified using a competitive binding ELISA kit (R\&D Systems) according to the manufacturer's protocol. Cell medium was centrifuged to remove particulates and the supernatant was diluted threefold before the assay. The plate was read with plate reader VersaMaxPLUS ROM v1.21 with SoftMax Pro v5.3b12 software at the absorbance of $450 \mathrm{~nm}$ with wavelength correction at $540 \mathrm{~nm}$ to correct for the optical imperfections in the 
plate. The concentration of PGE2 was calculated against a standard curve ranging from 0 to $2500 \mathrm{pg} / \mathrm{mL}$.

\section{ESE-1/Elf3 knockout mice and bone marrow-derived macrophage culture}

Elf3-/- mice on a C57BL/6 background were housed in pathogen-free condition at Toronto Centre for Phenogenomics (TCP), Toronto, Canada, and all procedures were approved by the Toronto Centre for Phenogenomics Animal Care Committee (Animal Use Protocol \#0062). Bone marrow was flushed from femur and tibia of Elf3-/- mice and wild-type littermates into single cell suspension and cultured in $20 \%$ L-929 conditioned media containing for 7 days as previously described [28]. Wild-type littermates were used as controls. The purity of bone marrow-derived macrophages was measured by flow cytometry with CD11b and F4/80 following methods from [10]. $4 \times 10^{5}$ of mature BMDMs were subjected to $100 \mathrm{ng} / \mathrm{mL}$ LPS or $10 \mathrm{ng} / \mathrm{mL}$ IL- 4 for $18 \mathrm{~h}$ to drive M1 and M2 polarization, respectively.

\section{Statistical analysis}

Statistical analysis was performed by two-tailed Student's $t$ test with Welch's corrections for unequal variances where appropriate, or by one-way paired ANOVA for multiple comparisons with Tukey's post-test or Bonferroni's post-test for selected pairs using GraphPad Prism 5.03 (GraphPad Software, Inc., La Jolla, CA, USA). P values of less than 0.05 were considered statistically significant.

\section{Results}

\section{ESE- 1 and COX- 2 are induced by IL- $1 \beta$ in human RASFs}

ESE-1 was previously shown to be rapidly upregulated by proinflammatory stimuli in human RASFs and maintained up to $24 \mathrm{~h}[15,16]$. To investigate its relationship to COX-2 expression, we stimulated primary RASFs with $10 \mathrm{ng} / \mathrm{mL}$ human IL-1 $\beta$ and quantified the amount of ESE-1 and COX-2 mRNA by RT-qPCR (Fig. 1a). We observed that ESE-1 mRNA expression peaked around $6 \mathrm{~h}$ and was reduced slightly at $24 \mathrm{~h}$. Transcriptional levels of COX-2, on the other hand, showed gradual increase until $6 \mathrm{~h}$ and downregulation at $24 \mathrm{~h}$ post IL-1 $\beta$ stimulation. Protein levels of ESE-1 varied among RA patients, though a representative is shown in Fig. 1c, indicating heterogeneity of the patient population and potentially the presence of other factors which may modulate ESE-1 expression (Additional file 2: Figure S1A). COX-2 protein, however, accumulated over time in most patients as previously shown [29] (Additional file 2: Figure S2B), which correlated with increase in $\mathrm{PGE}_{2}$ concentration (Fig. 1b).
RASFs can be effectively transduced by helper-dependent adenovirus (HD-Ad)

Helper-dependent adenovirus (HD-Ad) provide an attractive alternative means of gene delivery to non-viral vectors or other virus types, by its high carrying capacity of $37 \mathrm{~kb}$ and low immunogenicity from having all its viral coding sequences removed [20, 21]. HD-Ads have been successfully produced and used in our laboratory as a potential tool for cystic fibrosis (CF) gene therapy [30], as well as a research tool to knockdown ESE-1 in a number of studies $[23,24]$. However, similar to other adenoviruses, HD-Ad requires specific receptors to mediate viral attachment and gene transfer, notably the coxsackie virus and adenovirus receptor (CAR), which the fibroblasts are known to be lacking [31]. As expected, transduction with virus alone in human RASFs proved ineffective irrespective of viral dose (Fig. 2a), despite yielding close to $100 \%$ transduction in A549 lung adenocarcinoma cell line (data not shown). Non-covalent complexing of recombinant adenovirus with cationic molecules, however, has been demonstrated to significantly increase viral attachment and thus the efficiency of gene transfer by neutralizing the net negative surface charge on virus particles and the cell membrane [25, 32]. In an attempt to optimize viral infection in human RASFs, therefore, we complexed HD-Ad with DEAE-Dextran, which resulted in $100 \%$ cells being transduced at $100 \mathrm{MOI}$, with the expression lasting for more than $96 \mathrm{~h}$. We were able to achieve up to $90 \%$ knockdown of ESE-1 with HD-Ad carrying shESE-1 construct using this infection protocol (Fig. 2b). Although it was inevitable that the virus led to some degree of inflammation and ESE-1 activation, by $72 \mathrm{~h}$ from the initial exposure to virus particles, the background $\mathrm{PGE}_{2}$ and $\mathrm{COX}-2$ expression were comparable to basal levels prior to IL-1 $\beta$ stimulation. There was also minimal cell toxicity, if any, conferring a significant advantage over transfection. This indicates that with optimization with charge-neutralizing polymers such as DEAE-Dextran, HD-Ad can be an effective gene delivery tool for hard-to-transduce cell types such as immune cells and fibroblasts, and to study immune-responsive or cell survival genes that can be affected by transfection.

\section{Knockdown of ESE-1 leads to upregulation of COX-2 and increased $\mathrm{PGE}_{2}$ production}

In all patient RASF studied, knockdown of ESE-1 led to a significant upregulation of COX-2 at both RNA (Fig. 2b) and protein (Fig. 2d) levels. This correlated with increased concentration of $\mathrm{PGE}_{2}$ in the cell media (Fig. 2c), indicating functional significance of ESE-1 on COX-2 activity. There was also a recognizable heterogeneity in different patient RASFs resulting in different basal expression of ESE-1 following HD-Ad transduction 


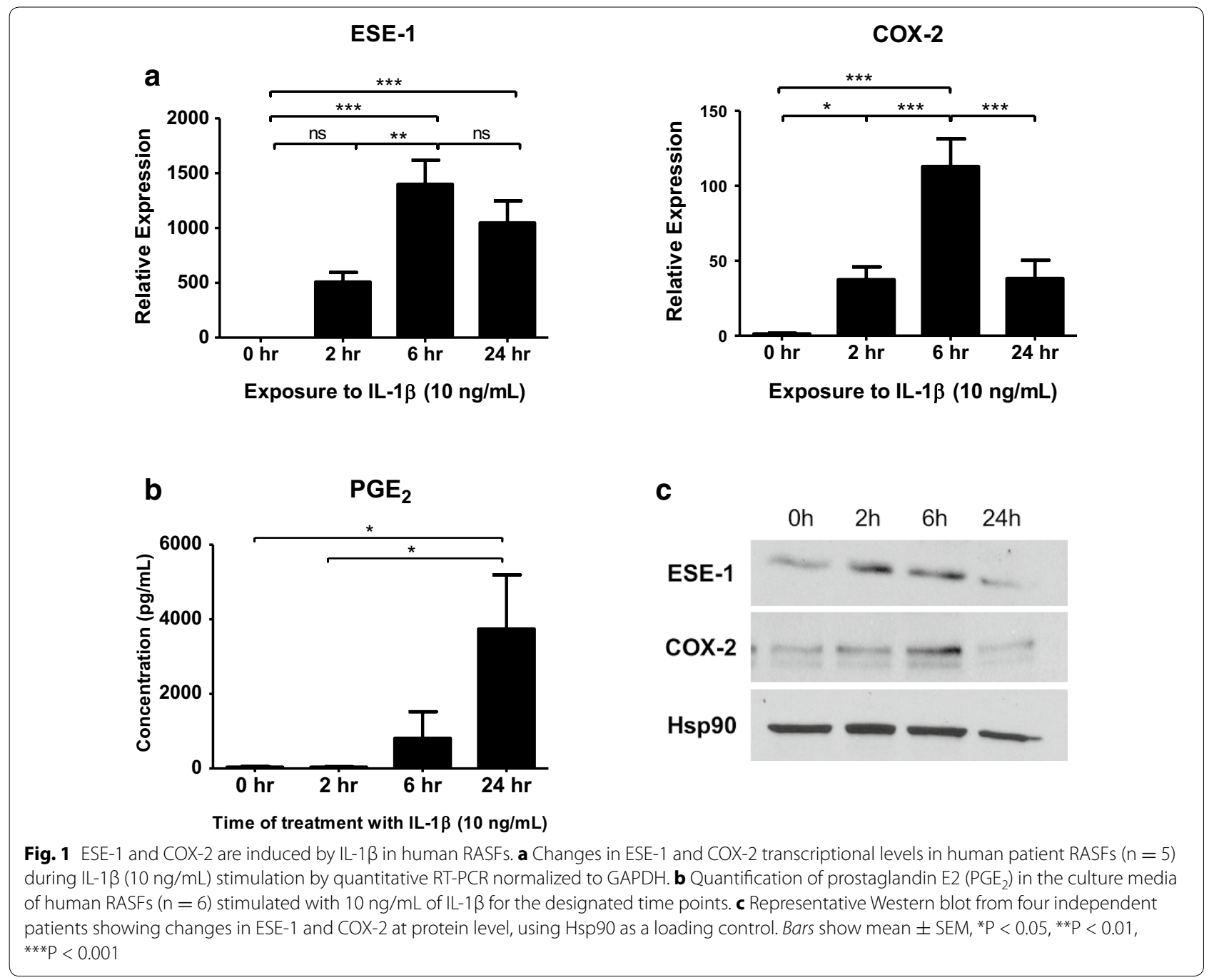

(Additional file 2: Figure 1c), but all showed similar trends of upregulated COX-2 following ESE-1 knockdown. Interestingly, however, ESE-1 knockdown had no effect on COX-2 basal level of mRNA expression or early phase of induction, and the effect of ESE-1 knockdown on COX was only visible at 24-hour time point (Fig. 2e). Similarly, while adenovirus binding to cultured synoviocytes is known to trigger COX-2 expression through the MAPK pathway, this subsides by $24 \mathrm{~h}$ [33] and in our study, transduced cells were stimulated with IL-1 $\beta 72 \mathrm{~h}$ after initial exposure to the virus when both COX-2 and $\mathrm{PGE}_{2}$ were comparable to the basal levels, thus minimizing confounding results from the inflammatory reaction to the viral vector. Still, C4HSU empty vector control virus was used in all experiments to account for the basal inflammatory response to the viral vector itself. Additionally, knockdown of ESE-1 had no effect on metalloprotease activity in RASFs, as shown by insignificant changes in MMP-1 or -13 mRNA expression (Additional file 2: Figure S2), indicating that ESE-1 may be a specific effector for resolving inflammatory responses.

\section{ESE-1 is localized in the nucleus}

ESE-1 was previously detected in the cytoplasm by immunostaining of RA patient tissue sections [16]. However, cytoplasmic/nuclear fractionation of activated human RASFs in in vitro following $24 \mathrm{~h}$ IL- $1 \beta$ stimulation revealed nuclear presence of ESE-1 (Fig. 3a, b), which was also consistent following shESE-1 HD-Ad viral transduction, where nuclear decrease in ESE-1 led to cytoplasmic increase in COX-2 (Fig. 3d). Furthermore, despite findings of ESE-1 cooperating with NFKB to transactivate target genes such as $i N O S$ in endothelial cells [34] and $C O X-2$ in macrophages [17], NFKB was localized in the cytoplasm by $24 \mathrm{~h}$ post IL- $1 \beta$ stimulation in RASFs (Fig. 3c, d). This was consistent with the previous finding where NFkB activated by IL- $1 \beta$ in RASFs resolved and returned to normal levels by $4 \mathrm{~h}$ of IL-1 $\beta$ stimulation [29]. 
a
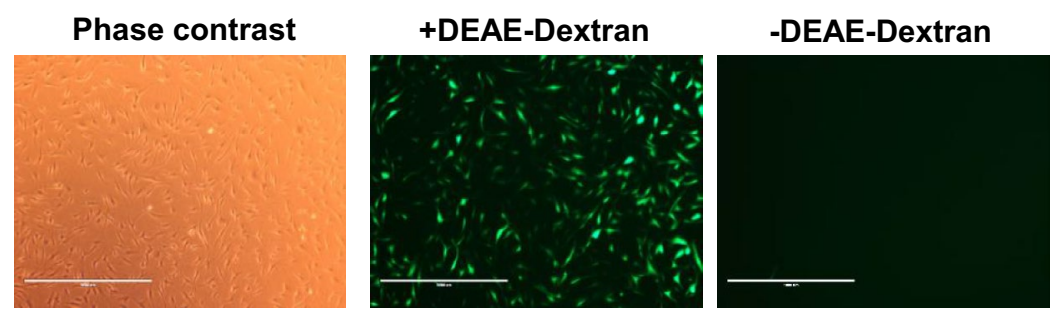

ESE-1

b

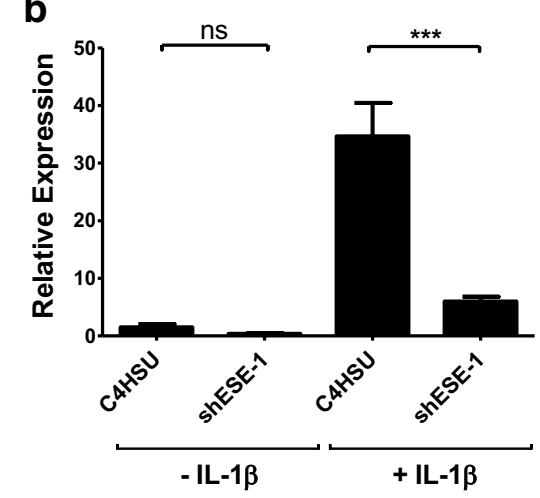

COX-2
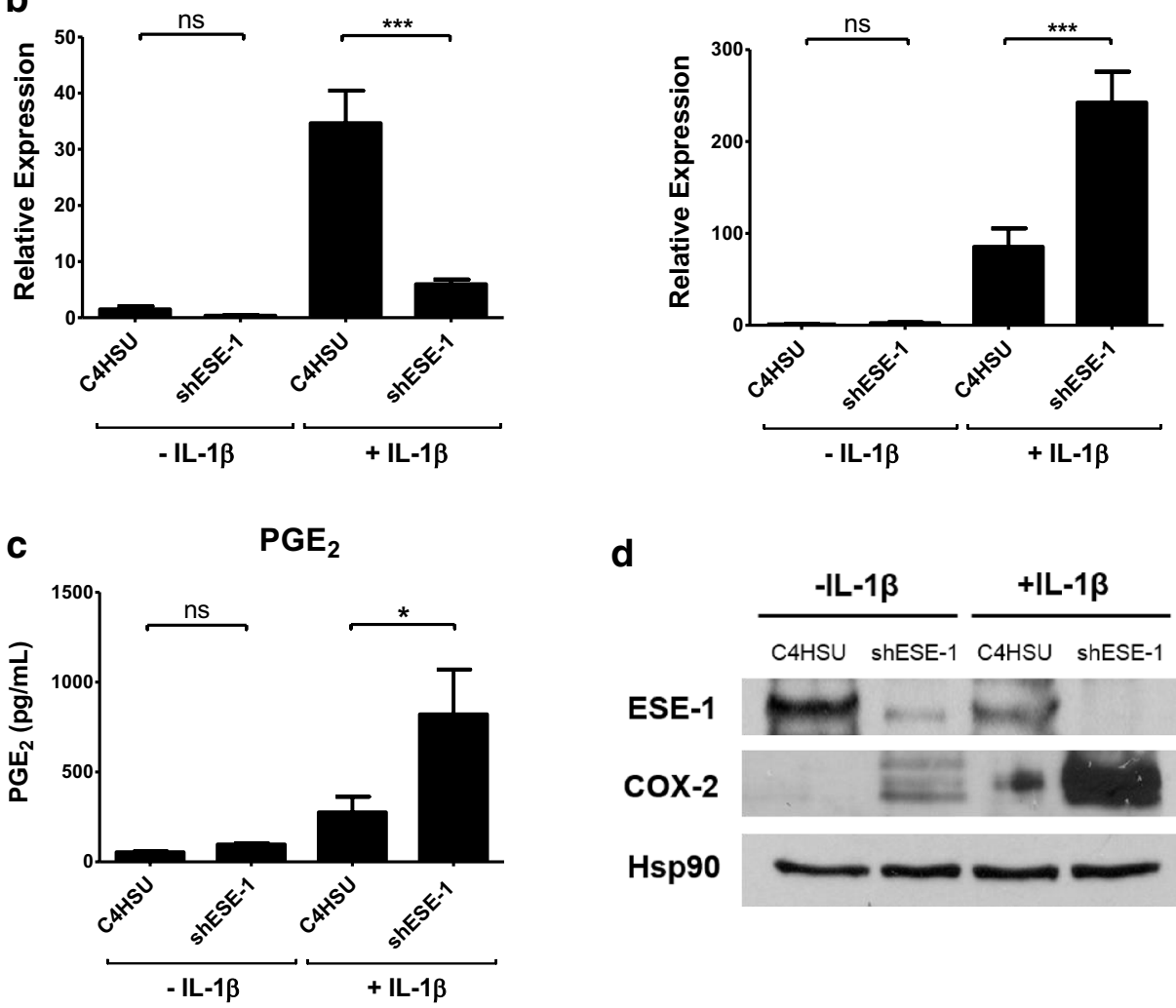

d

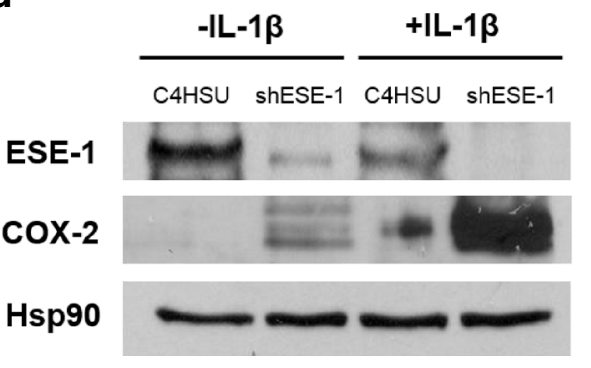

e

ESE-1
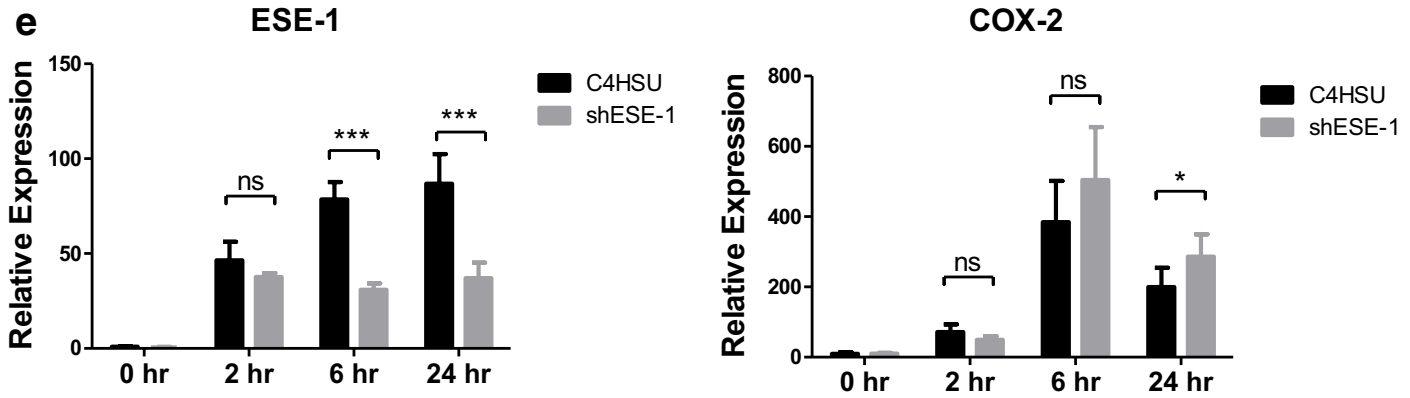

Fig. 2 Knockdown of ESE-1 leads to increased COX-2 and PGE 2 production in RASF. a Representative picture of human RASFs successfully transduced with helper-dependent adenovirus containing EGFP construct (HD-Ad-EGFP) with and without DEAE-Dextran. b Knockdown of ESE-1 leads to the transcriptional upregulation of COX-2 following $24 \mathrm{~h}$ of IL-1 $\beta$ stimulation $(n=6)$. The cells were incubated in the presence of HD-Ad and DEAE-Dextran complex for $48 \mathrm{~h}$ and starved in $0.5 \%$ FBS DMEM for $24 \mathrm{~h}$ prior to incubation with IL-1 $\beta$. Scale bar shows $100 \mu \mathrm{m}$. cTranscriptional upregulation of COX-2 is accompanied by increased production of $\mathrm{PGE}_{2}(n=6)$. $\mathbf{d}$ Representative Western blot showing changes in ESE-1 and COX-2 proteins in RASFs transduced with control (C4HSU) or shESE-1 HD-Ad. Bars show the mean $\pm \mathrm{SEM},{ }^{*} \mathrm{P}<0.05,{ }^{* * P}<0.01$, ${ }^{* * *} \mathrm{P}<0.001$, by one-way ANOVA Bonferroni's post-test. (E) Time point analysis of ESE-1 and COX-2 transcriptional levels $(n=5)$ during IL-1 $\beta$ stimulation after C4HSU or shESE-1 HD-Ad viral transduction. ns not significant, ${ }^{*} P<0.05$, ${ }^{* * *} P<0.001$ 
a

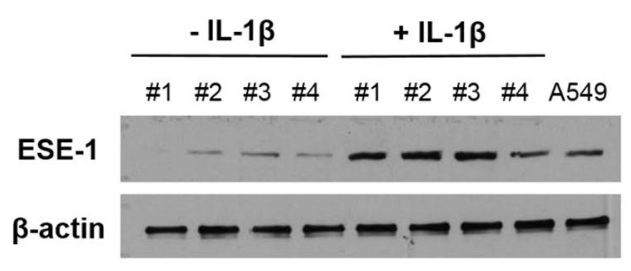

C

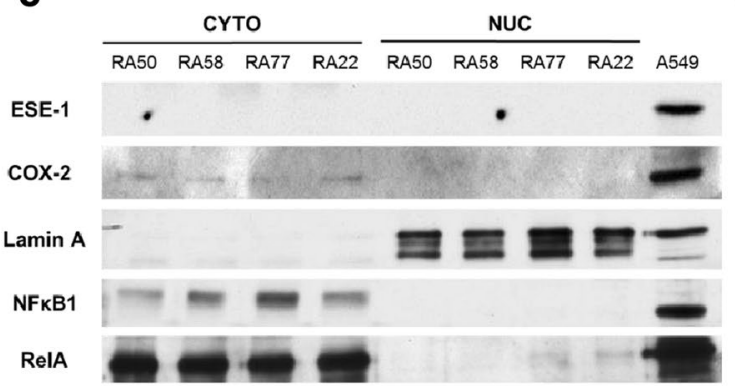

b

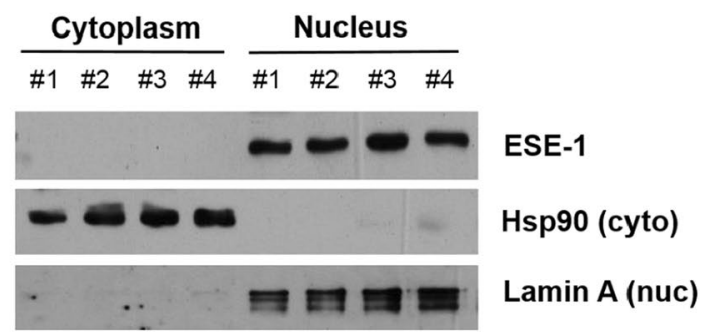

d

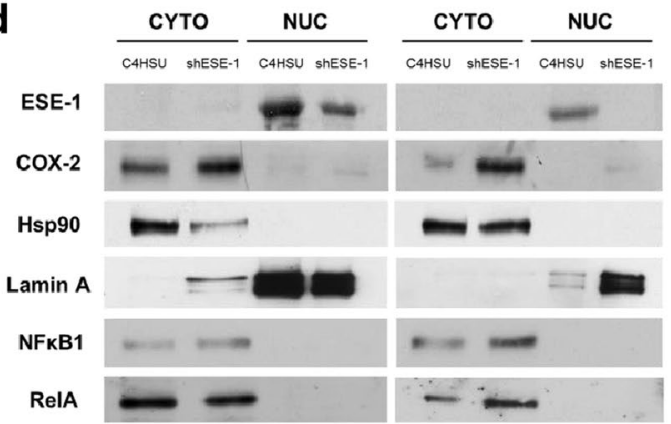

Fig. 3 ESE-1 is expressed in the nucleus of RASFs. a ESE-1 protein level increases with IL-1 $\beta$ stimulation $(n=4)$, shown by Western blot on whole cell lysates (WCLs) of stimulated or unstimulated RASFs. \#1-\#4 denotes patients \#50,58,77, and 22, and A549 lysate was included as positive control for ESE-1 protein. $\mathbf{b}$ ESE-1 protein is exclusively expressed in the nucleus, with $\mathrm{Hsp} 90$ and Lamin A as cytoplasmic and nuclear markers, respectively. Western blot of nuclear fractionated RASFs stimulated with $10 \mathrm{ng} / \mathrm{mL} \mathrm{IL-1 \beta}$ for $24 \mathrm{~h}$. c ESE-1 and COX-2 are minimally present in RASFs without IL-1 $\beta$ stimulation. d Knockdown of ESE-1 by HD-Ad-shESE-1 leads to increase in COX-2 expression in the cytoplasm in IL-1 $\beta$ treatment in RASFs compared to C4HSU control vector. Western blot showing results from two different patient RASFs

Therefore while NFkB may be responsible for the transcriptional upregulation of ESE-1 [23], it seems unlikely that NFKB is involved at the $24 \mathrm{~h}$ time point when ESE-1 knockdown enhances COX-2 expression. Nuclear localization of ESE-1 is in alignment with its known function as a transcription factor, and it may regulate other genes implicated in COX-2 regulation.

\section{SW982 cell line shows different pattern of ESE-1 expression from human primary RASFs}

The transcriptional expression pattern of ESE-1 in response to IL-1 $\beta$ has been studied in numerous nonepithelial cell lines, including human chondrocytes $(\mathrm{T} /$ C28a2, C28/I2, and C20A4), osteoblasts (LB-12), monocytes (THP-1), gliomas (U-138 MG and U-373 MG), and endothelial cells (HUVECs), where ESE-1 was shown to be one of the few Ets factors that were specifically responsive to IL-1 $\beta$-mediated activation, with typical induction pattern of peak expression between 2-6 $\mathrm{h}$ and dissipation by $24 \mathrm{~h}$ in most cell types [15-17, 34]. Studies have shown that SW982 synovial sarcoma cell line is representative of human primary synovial fibroblasts $[35,36]$. However, the expression pattern of ESE-1 in SW982, where ESE-1 peaked at $2 \mathrm{~h}$ and underwent drastic downregulation at $24 \mathrm{~h}$ (Fig. 4a), and protein expression peaking at $6 \mathrm{~h}$ and subsequently undergoing degradation (Fig. 4b), was different from that of RASFs, indicating that it is not a good representative cell line for our purposes. The knockdown of ESE-1 still had a visible effect on COX-2 upregulation at only $24 \mathrm{~h}$ (Fig. 4c), when ESE-1 protein was minimally present. p65 (= RelA) also dissipated by $24 \mathrm{~h}$ of IL- $1 \beta$, which made the performance of ChIP very difficult (data not shown). Therefore, it is possible that ESE-1 plays an indirect role or has other unknown function in COX-2 expression, such as mRNA stability, not just acting as a transcription factor, and the function of ESE-1 as a transcript, for example as a competing endogenous RNA, has never been explored.

\section{ESE-1/Elf3 knockout (KO) macrophages also show increased COX-2 mRNA expression}

ESE-1 was previously shown to modulate COX-2 in RAW267.4 cells [17]. Therefore, to address the difference in cell type, we also examined COX-2 levels in ESE-1/Elf3 knockout (KO) bone marrow-derived macrophages (BMDMs). Use of Elf3 KO BMDMs circumvented having to expose cells to additional inflammation from transfection- or transduction-mediated gene manipulation. In vitro differentiated BMDMs by CD11b and $\mathrm{F} 4 / 80$ staining were almost $100 \%$ pure (Fig. 5a), and Elf3 KO BMDMs showed no defect in macrophage differentiation which could affect its function [28], 

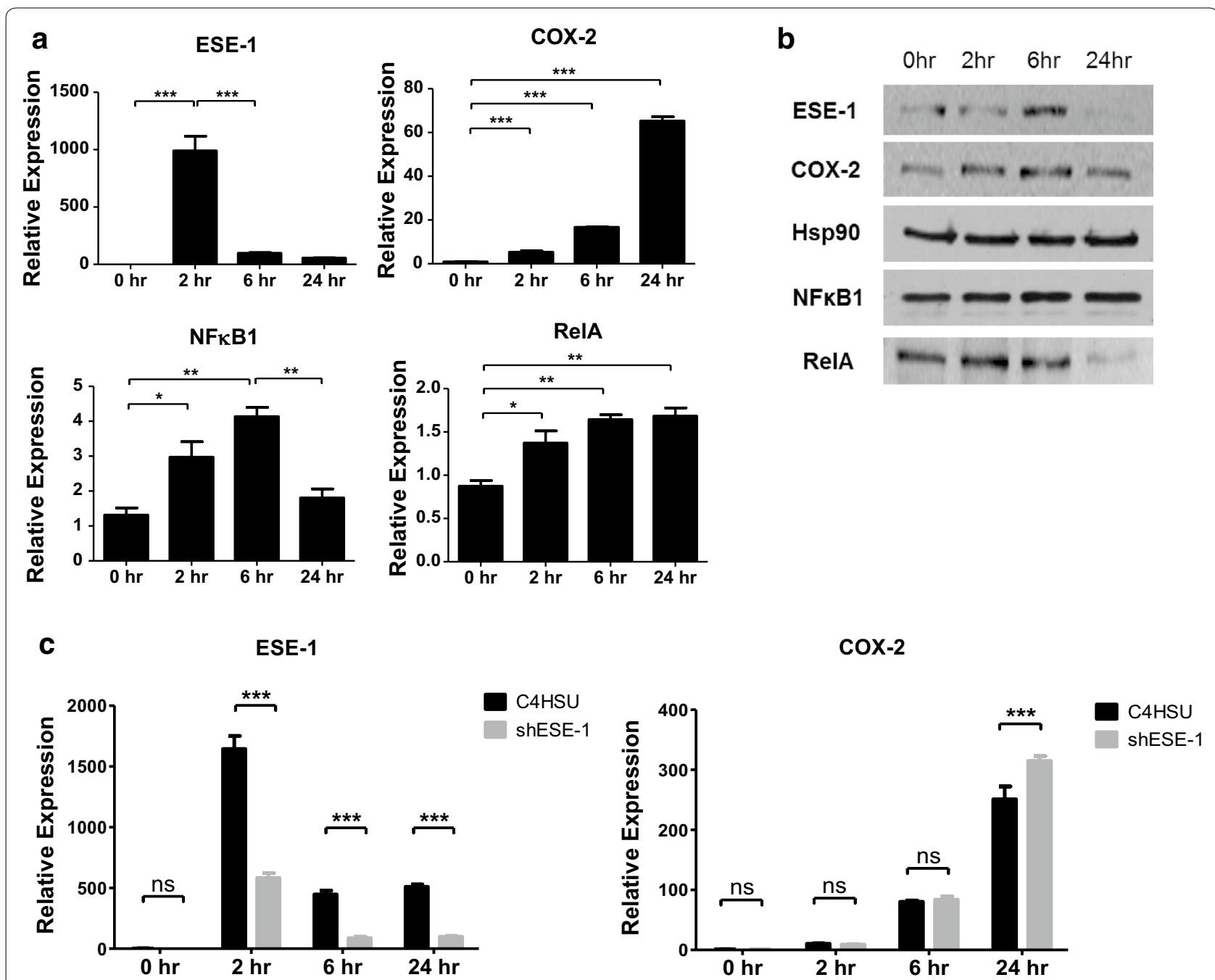

Fig. 4 ESE-1 downregulation leads to increased COX-2 mRNA in SW982 cell line. a Analysis of ESE-1, COX-2, RelA and NFkB1 transcriptional levels by RT-qPCR in SW982 cells during $\mathrm{LL}-1 \beta(10 \mathrm{ng} / \mathrm{mL})$ stimulation. b Representative Western blot of SW982 stimulated with $\mathrm{IL}-1 \beta(10 \mathrm{ng} / \mathrm{mL})$ over the $24 \mathrm{~h}$ time period. c Time course analysis of SW982 cells transduced with C4HSU or shESE-1 HD-Ad vectors and stimulated with IL-1 13 over $24 \mathrm{~h}$, started $72 \mathrm{~h}$ post-transduction. ${ }^{*} \mathrm{P}<0.05$, ${ }^{* *} \mathrm{P}<0.01$, ${ }^{* *} \mathrm{P}<0.001$, by one-way ANOVA Bonferroni's post-test, data shown are representative of two independent experiments with $n=3$ each

expressing normal levels of M-CSFR, Ly-6G, and Ly-6C (data not shown). When subjected to LPS, however, Elf3 KO BMDMs showed increased transcriptional level of COX-2 (Fig. 5b). There are two discrete classes of macrophages, namely M1, which is proinflammatory or classically activated, and M2, which is alternatively activated and takes on more immune-regulatory role. Classically activated M1 macrophages have increased production of pro-inflammatory cytokines such as TNF- $\alpha$ and IL-12, IL-23, nitric oxide (NO), and reactive oxygen species (ROS) and have increased antigen presentation and microbicidal activity, while M2 macrophages typically produce anti-inflammatory cytokines such as IL-10, IL-1 receptor antagonist (IL-1r $\alpha$ ) and promote tissue remodeling and repair [37]. Because COX-2 is one of the hallmark genes of M1 [37, 38], we also checked other genes related to M1- versus M2-polariation and observed that Elf3 KO BMDMs were slightly skewed towards M1 (Fig. 5c, d) by polarization-specific gene expression. A single knockout of Elf3 out of almost 30 Ets factors, however, may not show a dramatic effect due to compensation from other Ets, which adds complexity. Elf3 KO BMDMs in fact tended to have more Peas3 and Elf5 and KO lungs expressed higher levels of Erm (data not shown). Nevertheless, it is first time showing ESE-1 can have anti-inflammatory 


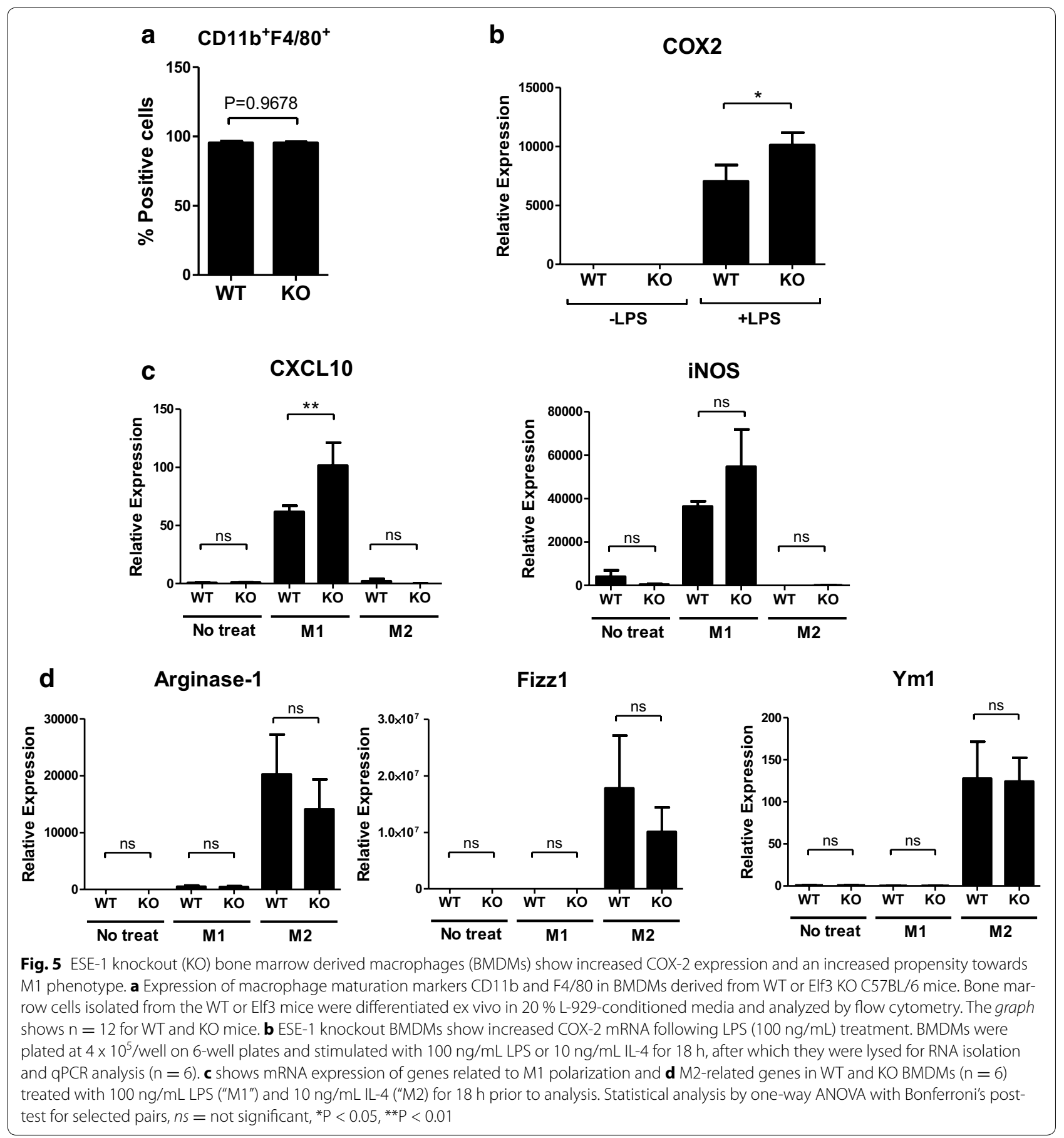

role in macrophages which may be subjected to further analysis.

\section{Discussion}

It is undisputable that understanding the pathogenesis of RA is critical for its prevention and treatment. However, persistent inflammation arises not only from persistent elicitation but also from incomplete resolution, and in pursuit of finding causative mechanisms, primary focus on effectors of pro-inflammatory response may have left some effectors playing dual or complex roles unrecognized from experimental approaches chosen to demonstrate one relationship but not the other. Our study illustrates one such example 
with an Ets transcription factor, ESE-1. ESE-1 was previously shown to co-operate with $\mathrm{NFKB}$ and positively regulate COX-2 by binding to the Ets-binding site on the COX-2 promoter [17]. However, by gene knockdown approach, we made an opposite observation in human RASFs, where knockdown of ESE-1 led to an upregulation of COX-2, which correlated with increased levels of $\mathrm{PGE}_{2}$. The use of HD-Ad-mediated knockdown is advantageous over previously employed conventional transfections, given its higher efficacy of gene delivery and long-term expression, as well as much lower cellular toxicity and immunogenicity.

It is important to note that previous findings on ESE-1 have been based on overexpression studies, but with insufficient consideration on the effect of the transfection procedure itself. Ectopic gene expression can lead to supraphysiological levels of the gene of interest, as well as cell toxicity from the transfection. Also, overexpression by transfection may not accurately reflect the temporal behavior of a protein, and may thus lead to artificial interaction or co-localization of proteins that normally do not co-exist under physiological conditions. For example, in RASFs, NFkB is resolved within $4 \mathrm{~h}$ following IL- $1 \beta$ stimulation [29], yet the effect of ESE-1 knockdown was only evident at $24 \mathrm{~h}$ time point, when the initial inflammation induced by IL- $1 \beta$ had mostly resolved and ESE- 1 and $\mathrm{NF}_{\kappa} \mathrm{B}$ were disparately localized in the nucleus and cytoplasm, respectively. Also, knockdown of ESE-1 had no effect on COX-2 induction in RASF or SW982, indicating that ESE-1 may not play a direct role in regulating COX-2 transcription as previously thought. Rather, given that $\mathrm{PGE}_{2}$ has been shown to prolong COX-2 mRNA half-life through the p38 MAPK pathway, ESE-1 may be functioning more as a downstream effector of $\mathrm{PGE}_{2}$ signaling than IL- $1 \beta$ at the $24-\mathrm{h}$ time point. In fact, transcriptional activation of COX-2 in NIH $3 \mathrm{~T} 3$ fibroblasts by $\mathrm{PGE}_{2}$ was found to require $\mathrm{C} / \mathrm{EBP}$ and CRE-1 sites but not NFKB [39], suggesting that ESE-1 may be interacting with protein partners other than $\mathrm{NF \kappa B}$ or assuming other functions at later time points.

COX-2 regulation is complex, and occurs at both transcriptional and non-transcriptional levels. The exact transcription factor complexes that are recruited at the COX-2 promoter site vary by cell type and stimulation [40]. Sequence analysis of the $5^{\prime}$-flanking region of the human COX-2 gene has identified several potential transcriptional regulatory elements, including two nuclear factor kappa B (NF-kB) sites, an SP1 site, a CAAT enhancer binding protein $(\mathrm{C} / \mathrm{EBP})$, nuclear factor for interleukin-6 expression (NF-IL6) motif, two AP-2 sites, an E-box, and a TATA-box, as well as a peroxisome proliferator response element (PPRE), two cyclic AMP response elements (CRE), and a sterol response element
(SRE) [41]. Additionally, COX-2 can be regulated posttranscriptionally by its mRNA stability with the involvement of molecules such as HuR, microRNA 101a and 199a and alternative polyadenylation [42] and by longnoncoding RNAs [43], as well as at its protein level by $\mathrm{N}$-glycosylation at Asn-594 and by substrate-dependent suicide inactivation [44]. Therefore, elucidation of the exact role of ESE-1 in COX-2 regulation warrants a separate study.

However, one possible mechanism by which ESE-1 exerts its repressor function on COX-2 transcription may be through ESE-3, another closely related Ets factor and a direct target gene of ESE-1. Unlike ESE-1 which typically peaks at $2 \mathrm{~h}$ following cytokine stimulation, ESE-3 was found to peak around $24 \mathrm{~h}$ in human airway epithelial cells [23]. While Wu et al. did not consider the possibility of ESE-3 acting as a reciprocal repressor of ESE-1, their data indicates that overexpression of ESE-3 was in fact more effective in inhibiting ESE-1 transcription than ESE-1 itself [23]. Similarly, p38 MAPK plays a crucial role in prolonging COX-2 mRNA stability by $\mathrm{PGE}_{2}$ $[45,46]$, and ESE-3 is known to act as a downstream repressor of p38 MAPK pathway under certain conditions [47]. Therefore, it is possible that ESE-3 acts as a reciprocal repressor of ESE-1 at later time point when $\mathrm{IL}-1 \beta$ is degraded, and that this feedback loop is defective in RASFs. Given that ESE-3 is also upregulated during stress-induced senescence in human fibroblasts [48], dysregulation in the ESE-1-ESE-3-MAPK regulatory loop may be involved in sustaining the non-senescent phenotype of RASFs.

Lastly, although knockdown of ESE-1 also resulted in upregulation of COX-2 in SW982 cells, the pattern of ESE-1 expression induced by IL- $1 \beta$ was very different, indicating SW982 is not a truly representative model to study the role ESE-1 in human RASFs in vitro. Because most of the ESE-1 targets have been identified in immortalized cell lines, this points to the need to develop better cell model systems that more closely mimic RASFs in situ, as well as experimental tools which minimally interfere with immune-responsive function of the target proteins. Nonetheless, our findings reveal new insights into the role of ESE-1 in rheumatoid arthritis, as it is the first time to demonstrate that ESE-1 may assume an anti-inflammatory role under physiological conditions to prevent excessive tissue damage during an inflammatory response, by negatively regulating COX-2 in human RASFs.

\section{Conclusions}

ESE-1 acts as a negative regulator of COX-2 in human RASFs and its effect on COX-2 is NFkB-independent, occurring at later phases of an inflammatory response. 
This may indicate that ESE-1 is involved in the resolution of inflammation unlike previously thought, and this discrepancy may be attributed to confounding responses to transfection procedures.

\section{Additional files}

\author{
Additional file 1: Table S1. RT-qPCR primer sequences used in the \\ study.
}

Additional file 2: Figure S1. Additional patient data for Figure 1 and Figure 2.

Additional file 3: Figure S2. $Q P C R$ analysis of ESE-1 knockdown in human RASFs $(n=6)$ for (A) MMP-1 and MMP-13, and (B) NFkB1 and RelA. $\mathrm{ns}=$ not significant.

\begin{abstract}
Abbreviations
AA: arachidonic acid; AP-1: activator protein 1; BMDM: bone marrow-derived macrophage; C4HSU: control helper-dependent adenoviral vector; cDNA: complementary deoxyribonucleic acid; C/EBP: CAAT enhancer binding protein; COX-2: cyclooxygenase-2; CRE: cyclic AMP response elements; DEAE-Dextran: diethylaminoethyl-dextran; DMEM: Dulbecco's Modified Eagle Medium; Elf3: E74-like factor 3; ELISA: enzyme-linked immunosorbent assay; ESE-1: epithelium-specific Ets transcription factor-1; Ets: E26 transformationspecific domain; FBS: fetal bovine serum; GAPDH: glyceraldehyde-3-phosphate dehydrogenase; HD-Ad: helper-dependent adenovirus; Hsp90: heat shock protein 90; IL-1 $\beta$ : interleukin-1 $\beta$; KO: knockout; LPS: lipopolysaccharide; MAPK: mitogen-activated protein kinase; MMP: matrix metalloproteinase; MOI: multiplicity of infection; mRNA: messenger RNA; NF-IL6: nuclear factor for interleukin-6 expression motif; NFkB: nuclear factor kappa B; NO: nitric oxide; NSAIDs: non-steroidal anti-inflammatory drugs; PG: prostaglandin; $\mathrm{PGE}_{2}$ : prostaglandin $\mathrm{E}_{2} ; \mathrm{PGH}_{2}$ : prostaglandin $\mathrm{H}_{2} ;$ PPRE: peroxisome proliferator response element; RA: rheumatoid arthritis; RASF: rheumatoid arthritis synovial fibroblast; RNA: ribonucleic acid; shRNA: short-hairpin RNA; ROS: reactive oxygen species; RPMI: Roswell Park Memorial Institute medium; RT-qPCR: quantitative reverse transcription polymerase chain reaction; SRE: sterol response element; TCP: Toronto Centre for Phenogenomics; TNF-a: tumor necrosis factor-a; WT: wild-type.
\end{abstract}

\section{Authors' contributions}

$\mathrm{CL}$ carried out the in vitro treatments and RNA quantifications, and drafted the manuscript. SG performed the cytoplasmic-nuclear fractionations and densitometry, and helped to draft the manuscript. JW carried out the Western blots and statistical analyses, and helped to revise the manuscript. EJ was involved in acquiring patient synovial tissues and extracting synovial fibroblasts for the experiments, and revised the manuscript. LC participated in the study design and critically revising the manuscript. JM provided input on data analyses, and edited the manuscript. MK participated in the study design and its coordination and data interpretation, and helped to draft the manuscript. JH conceived the study, participated in the optimization of the viral transduction, and edited the manuscript. All authors read and approved the final manuscript.

\section{Author details}

${ }^{1}$ SickKids Research Institute, Program in Physiology and Experimental Medicine, The Hospital for Sick Children, Peter Gilgan Centre for Research and Learning, 9th floor, 686 Bay Street, Toronto, ON M5G 0A4, Canada. ${ }^{2}$ Laboratory Medicine and Pathobiology, University of Toronto, 1 King's College Circle, Toronto, ON M5S 1A8, Canada. ${ }^{3}$ The Keenan Research Centre, Li Ka Shing Knowledge Institute, St. Michael's Hospital, 209 Victoria Street, Toronto, ON M5B 1T8, Canada. ${ }^{4}$ Institute of Medical Science, Faculty of Medicine, University of Toronto, 1 King's College Circle, Toronto, ON M5S 1A8, Canada. ${ }^{5}$ Department of Surgery, St. Michael's Hospital, University of Toronto, 30 Bond Street, Toronto, ON M5B 1W8, Canada. ${ }^{6}$ Department of Anesthesiology and Intensive Care, The Second Military Medical University, Changhai Hospital, Shanghai 200433, China. ${ }^{7}$ Department of Medicine, Division of Rheumatology and Immunology, School of Medicine, Vanderbilt University, 116121 st Ave S, MCN T-3113, Nashville, TN 37232, USA. ${ }^{8}$ Division of Genetics and Development, Toronto Western Research Institute, Toronto Western Hospital, University Health Network (UHN), 60 Leonard Avenue, Toronto, ON M5T 2S8, Canada.

\section{Acknowledgements}

We would like to thank Ms. Jing Wu and Ms. Cathleen Duan for the production of C4HSU and shESE-1 helper-dependent adenoviruses.

\section{Competing interests}

The authors declare that they have no competing interests.

\section{Funding}

This study was funded by Canadian Institute for Health Research (CIHR) Grant (MOP 125882) to Jim Hu. Chan Mi Lee is a recipient of Queen Elizabeth II/ Canadian Arthritis Network Scholarships in Science and Technology from University of Toronto and SickKids Restracomp Studentship from SickKids Hospital.

Received: 19 April 2016 Accepted: 25 May 2016

Published online: 16 June 2016

\section{References}

1. McInnes IB, Schett G. Cytokines in the pathogenesis of rheumatoid arthritis. Nat Rev Immunol. 2007;7:429-42.

2. Fattahi MJ, Mirshafiey A. Prostaglandins and rheumatoid arthritis. Arthritis. 2012;2012:239310.

3. Ricciotti E, FitzGerald GA. Prostaglandins and inflammation. Arterioscler Thromb Vasc Biol. 2011;31(5):986-1000.

4. Crofford LJ, Wilder RL, Ristimaki AP, Sano H, Remmers EF, Epps HR, Hla T. Cyclooxygenase-1 and -2 expression in rheumatoid synovial tissues. Effects of interleukin-1 beta, phorbol ester, and corticosteroids. J Clin Investig. 1994;93:1095-101.

5. Siegle I, Klein T, Backman JT, Saal JG, Nusing RM, Fritz P. Expression of cylooxygenase 1 and cyclooxygenase 2 in human synovial tissue: differential elevation of cyclooxygenase 2 in inflammatory joint diseases. Arthritis Rheum. 1998:41:122-9.

6. Martel-Pelletier J, Pelletier JP, Fahmi H. Cyclooxygenase-2 and prostaglandins in articular tissues. Semin Arthritis Rheum. 2003;33(3):155-67.

7. Seppala E, Nissila M, Isomaki H, Wuorela H, Vapaatalo H. Effects of nonsteroidal anti-inflammatory drugs and prednisolone on synovial fluid white cells, prostaglandin E2, leukotriene B4 and cyclic AMP in patients with rheumatoid arthritis. Scand J Rheumatol. 1990;19:71-5.

8. Flower RJ. The development of COX2 inhibitors. Nat Rev Drug Discov. 2003;2(3):179-91.

9. Appleby S, Ristimaki A, Neilson K, Narko K, Hla T. Structure of the human cyclo-oxygenase-2 gene. Biochem J. 1994:302:723-7.

10. Teruyama K, Abe M, Nakano T, Iwasaka-Yagi C, Takahashi S, Yamada S, Sato Y. Role of transcription factor Ets-1 in the apoptosis of human vascular endothelial cells. J Cell Physiol. 2001;188:243-52.

11. Howe LR, Crawford HC, Subbaramaiah K, Hassell JA, Dannenberg AJ, Brown AM. PEA3 is up-regulated in response to Wnt1 and activates the expression of cyclooxygenase-2. J Biol Chem. 2001;276:20108-15.

12. Joo M, Park GY, Wright JG, Blackwell TS, Atchison ML, Christman JW. Transcriptional regulation of the cyclooxygenase-2 gene in macrophages by PU.1. J Biol Chem. 2004;279(8):6658-65.

13. Zhang XF, Zhu Y, Liang WB, Zhang JJ. The ETS-domain transcription factor Elk-1 regulates COX-2 gene expression and inhibits glucose-stimulated insulin secretion in the pancreatic beta-cell line INS-1. Int J Endocrinol. 2013;2013:843462.

14. Oliver JR, Kushwah R, Hu J. Multiple roles of the epithelium-specific ETS transcription factor, ESE-1, in development and disease. Lab Invest. 2012;92(3):320-30,

15. Brown C, Gaspar J, Pettit A, Lee R, Gu X, Wang H, Manning C, Voland C, Goldring SR, Goldring MB, et al. ESE-1 is a novel transcriptional mediator of angiopoietin-1 expression in the setting of inflammation. J Biol Chem. 2004;279(13):12794-803.

16. Grall F, Gu X, Tan L, Cho JY, Inan MS, Pettit AR, Thamrongsak U, Choy BK, Manning C, Akbarali Y, et al. Responses to the proinflammatory 
cytokines interleukin-1 and tumor necrosis factor alpha in cells derived from rheumatoid synovium and other joint tissues involve nuclear factor kappaB-mediated induction of the Ets transcription factor ESE-1. Arthritis Rheum. 2003:48(5):1249-60

17. Grall FT, Prall WC, Wei W, Gu X, Cho JY, Choy BK, Zerbini LF, Inan MS, Goldring SR, Gravallese EM, et al. The Ets transcription factor ESE-1 mediates induction of the COX-2 gene by LPS in monocytes. FEBS $\mathrm{J}$ 2005;272(7):1676-87.

18. Neumann E, Lefevre S, Zimmermann B, Gay S, Muller-Ladner U. Rheumatoid arthritis progression mediated by activated synovial fibroblasts. Trends Mol Med. 2010;16(10):458-68.

19. Bartok B, Firestein GS. Fibroblast-like synoviocytes: key effector cells in rheumatoid arthritis. Immunol Rev. 2010;233(1):233-55.

20. Brunetti-Pierri N, Ng P. Helper-dependent adenoviral vectors. In: Chernajovsky Y, Robbins P, editors. Gene therapy for autoimmune and inflammatory diseases. Basel: Springer; 2010. p. 193-207.

21. Vetrini F, Ng P. Gene therapy with helper-dependent adenoviral vectors: current advances and future perspectives. Viruses. 2010;2(9):1886-917.

22. Lee CM, Gupta S, Parodo J, Wu J, Marshall JC, Hu J. The uncovering of ESE-1 in human neutrophils: implication of its role in neutrophil function and survival. Genes Immun. 2015:16(5):356-61.

23. Wu J, Duan R, Cao H, Field D, Newnham CM, Koehler DR, Zamel N, Pritchard MA, Hertzog P, Post M, et al. Regulation of epithelium-specific Ets-like factors ESE-1 and ESE-3 in airway epithelial cells: potential roles in airway inflammation. Cell Res. 2008;18(6):649-63.

24. Yu Z, Xu J, Liu J, Wu J, Lee CM, Yu L, Hu J. Epithelium-Specific Ets-Like Transcription Factor 1, ESE-1, Regulates ICAM-1 Expression in Cultured Lung Epithelial Cell Lines. Mediators Inflamm. 2015;2015:547928.

25. Fasbender A, Zabner J, Chillon M, Moninger TO, Puga AP, Davidson BL, Welsh MJ. Complexes of adenovirus with polycationic polymers and cationic lipids increase the efficiency of gene transfer in vitro and in vivo. J Biol Chem. 1997:272(10):6479-89.

26. Livak KJ, Schmittgen TD. Analysis of relative gene expression data using real-time quantitative PCR and the 2(-Delta Delta C(T)) Method. Methods. 2001;25(4):402-8.

27. Suzuki K, Bose P, Leong-Quong RY, Fujita DJ, Riabowol K. REAP: a two minute cell fractionation method. BMC Res Notes. 2010;3:294.

28. Lee $\mathrm{CM}$, Hu J. Cell density during differentiation can alter the phenotype of bone marrow-derived macrophages. Cell Biosci. 2013;3:30.

29. Crofford LJ, Tan B, McCarthy CJ, Hla T. Involvement of nuclear factor kappa $B$ in the regulation of cyclooxygenase-2 expression by interleukin-1 in rheumatoid synoviocytes. Arthritis Rheum. 1997:40(2):226-36.

30. Cao H, Machuca TN, Yeung JC, Wu J, Du K, Duan C, Hashimoto K, Linacre V, Coates AL, Leung K, et al. Efficient gene delivery to pig airway epithelia and submucosal glands using helper-dependent adenoviral vectors. Mol Ther Nucleic Acids. 2013;2:e127.

31. Hidaka C, Milano E, Leopold PL, Bergelson JM, Hackett NR, Finberg RW, Wickham TJ, Kovesdi I, Roelvink P, Crystal RG. CAR-dependent and CARindependent pathways of adenovirus vector-mediated gene transfer and expression in human fibroblasts. J Clin Invest. 1999;103(4):579-87.

32. Kushwah R, Oliver JR, Cao H, Hu J. Nacystelyn enhances adenoviral vector-mediated gene delivery to mouse airways. Gene Ther. 2007;14(16):1243-8.

33. Crofford LJ, McDonagh KT, Guo S, Mehta H, Bian H, Petruzelli LM, Roessler BJ. Adenovirus binding to cultured synoviocytes triggers signaling through MAPK pathways and induces expression of cyclooxygenase-2. Gene Med. 2005;7(3):288-96.
34. Rudders S, Gaspar J, Madore R, Voland C, Grall F, Patel A, Pellacani A, PerreIla MA, Libermann TA, Oettgen P. ESE-1 is a novel transcriptional mediator of inflammation that interacts with NF-kappa B to regulate the inducible nitric-oxide synthase gene. J Biol Chem. 2001;276(5):3302-9.

35. Chang JH, Lee KJ, Kim SK, Yoo DH, Kang TY. Validity of SW982 synovial cell line for studying the drugs against rheumatoid arthritis in fluvastatin-induced apoptosis signaling model. Indian J Med Res. 2014;139(1):117-24

36. Yamazaki T, Yokoyama T, Akatsu H, Tukiyama T, Tokiwa T. Phenotypic characterization of a human synovial sarcoma cell line, SW982, and its response to dexamethasone. In Vitro Cell Develop Biol Animal. 2003;39(8-9):337-9.

37. Mantovani A, Biswas SK, Galdiero MR, Sica A, Locati M. Macrophage plasticity and polarization in tissue repair and remodelling. J Pathol. 2013;229(2):176-85.

38. Martinez FO, Gordon S, Locati M, Mantovani A. Transcriptional profiling of the human monocyte-to-macrophage differentiation and polarization: new molecules and patterns of gene expression. J Immunol. 2006;177(10):7303-11.

39. Bagga D, Wang L, Farias-Eisner R, Glaspy JA, Reddy ST. Differential effects of prostaglandin derived from omega- 6 and omega-3 polyunsaturated fatty acids on COX-2 expression and IL-6 secretion. Proc Natl Acad Sci USA. 2003;100(4):1751-6.

40. Kang YJ, Mbonye UR, DeLong CJ, Wada M, Smith WL. Regulation of intracellular cyclooxygenase levels by gene transcription and protein degradation. Prog Lipid Res. 2007;46(2):108-25.

41. Tanabe T, Tohnai N. Cyclooxygenase isozymes and their gene structures and expression. Prostaglandins Other Lipid Mediat. 2002;68-69:95-114.

42. Harper KA, Tyson-Capper AJ. Complexity of COX-2 gene regulation. Biochem Soc Trans. 2008;36(Pt 3):543-5.

43. Krawczyk M, Emerson BM. p50-associated COX-2 extragenic RNA (PACER) activates COX-2 gene expression by occluding repressive NF-kappaB complexes. ELife. 2014;3:e01776.

44. Mbonye UR, Yuan C, Harris CE, Sidhu RS, Song I, Arakawa T, Smith WL. Two distinct pathways for cyclooxygenase-2 protein degradation. J Biol Chem. 2008;283(13):8611-23.

45. Dean JL, Brook M, Clark AR, Saklatvala J. p38 mitogen-activated protein kinase regulates cyclooxygenase-2 mRNA stability and transcription in lipopolysaccharide-treated human monocytes. J Biol Chem. 1999:274(1):264-9.

46. Lasa M, Mahtani KR, Finch A, Brewer G, Saklatvala J, Clark AR. Regulation of cyclooxygenase 2 mRNA stability by the mitogen-activated protein kinase p38 signaling cascade. Mol Cell Biol. 2000;20(12):4265-74.

47. Tugores A, Le J, Sorokina I, Snijders AJ, Duyao M, Reddy PS, Carlee L, Ronshaugen M, Mushegian A, Watanaskul T, et al. The epitheliumspecific ETS protein EHF/ESE-3 is a context-dependent transcriptional repressor downstream of MAPK signaling cascades. J Biol Chem. 2001;276(23):20397-406.

48. Fujikawa M, Katagiri T, Tugores A, Nakamura Y, Ishikawa F. ESE-3, an Ets family transcription factor, is up-regulated in cellular senescence. Cancer Sci. 2007;98(9):1468-75.

\section{Submit your next manuscript to BioMed Central and we will help you at every step:}

- We accept pre-submission inquiries

- Our selector tool helps you to find the most relevant journal

- We provide round the clock customer support

- Convenient online submission

- Thorough peer review

- Inclusion in PubMed and all major indexing services

- Maximum visibility for your research

Submit your manuscript at www.biomedcentral.com/submit 